\title{
Severe SARS-CoV-2 placenta infection can impact neonatal outcome in the absence of vertical transmission
}

\author{
Fulvia Milena Cribiù, ${ }^{1}$ Roberta Erra, ${ }^{1}$ Lorenza Pugni, ${ }^{1}$ Carlota Rubio-Perez, ${ }^{2}$ Lidia Alonso, ${ }^{2}$ Sara Simonetti, ${ }^{2}$ Giorgio Alberto Croci, ${ }^{1}$ \\ Garazi Serna, ${ }^{2}$ Andrea Ronchi, ${ }^{1}$ Carlo Pietrasanta, ${ }^{1}$ Giovanna Lunghi, ${ }^{1}$ Anna Maria Fagnani, ${ }^{1}$ Maria Piñana, ${ }^{3}$ Matthias Matter, ${ }^{4}$ \\ Alexandar Tzankov, ${ }^{4}$ Luigi Terracciano, ${ }^{4}$ Andres Anton, ${ }^{3}$ Enrico Ferrazzi, ${ }^{1}$ Stefano Ferrero, ${ }^{1}$ Enrico Iurlaro, ${ }^{1}$ Joan Seoane, ${ }^{2,5}$ \\ and Paolo Nuciforo² \\ 'Fondazione IRCCS Ca' Granda Ospedale Maggiore Policlinico, Milan, Italy. ${ }^{2}$ Vall d'Hebron Institute of Oncology, Barcelona, Spain. ${ }^{3}$ Vall d'Hebron University Hospital, Vall d'Hebron Institute of Research, \\ Barcelona, Spain. ${ }^{4}$ University of Basel, Basel, Switzerland. ${ }^{5}$ Institució Catalana de Recerca i Estudis Avançats (ICREA), Universitat Autonoma de Barcelona (UAB), CIBERONC, Barcelona, Spain.
}

The effect of severe acute respiratory syndrome coronavirus 2 (SARS-CoV-2) infection on the pathophysiology of the placenta and its impact on pregnancy outcome has not yet been fully elucidated. Here, we present a comprehensive clinical, morphological, and molecular analysis of placental tissues from pregnant women with and without SARSCoV-2 infection. SARS-CoV-2 could be detected in half of placental tissues from SARS-CoV-2-positive women. The presence of the virus was not associated with any distinctive pathological, maternal, or neonatal outcome features. SARS-CoV-2 tissue load was low in all but one patient who exhibited severe placental damage leading to neonatal neurological manifestations. The placental transcriptional response induced by high viral load of SARS-CoV-2 showed an immunopathology phenotype similar to autopsy lung tissues from patients with severe coronavirus disease 2019. This finding contrasted with the lack of inflammatory response in placental tissues from SARS-CoV-2-positive women with low viral tissue load and from SARS-CoV-2-negative women. Importantly, no evidence of vertical transmission of SARSCoV-2 was found in any newborns, suggesting that the placenta may be an effective maternal-neonatal barrier against the virus even in the presence of severe infection. Our observations suggest that severe placental damage induced by the virus may be detrimental for the neonate independently of vertical transmission.

\section{Introduction}

The low incidence of vertical transmission reported so far (1-8) suggest that the placenta may play a critical role in inhibiting intrauterine transmission of severe acute respiratory syndrome coronavirus 2 (SARS-CoV-2) to the fetus. Several recent individual case or cohort reports have shown that SARS-CoV-2 may reach the placenta (9-17). However, vertical transmission remains controversial and a cause-effect relationship between the pathophysiological response of the placenta according to disease severity and its association with neonatal outcome has not been formally proven.

\section{Results and Discussion}

Demographic and epidemiologic features of women included in the study and their outcomes at delivery are shown in Table 1 and Table 2. Among the 37 pregnant women of our cohort, 21 had confirmed SARS-CoV-2 infection by nasopharyngeal swab PCR testing. Median gestational age (weeks+days) was

Authorship note: FMC, RE, LP, JS, and PN contributed equally to this work. Conflict of interest: The authors have declared that no conflict of interest exists. Copyright: ( 2021, American Society for Clinical Investigation.

Submitted: October 23, 2020; Accepted: January 20, 2021; Published: March 15, 2021. Reference information: J Clin Invest. 2021;131(6):e145427. https://doi.org/10.1172/JCl145427.
$37+3$ in the cohort of patients positive for coronavirus disease 2019 (COVID-19+) and 39+1 in the COVID-19- cohort. The median time from SARS-CoV-2 infection diagnosis to delivery was 2 days (interquartile range, 1-6 days). All women successfully delivered live and asymptomatic newborns, except in 2 cases. One SARS-CoV-2-negative woman underwent labor induction for therapeutic miscarriage because of chromosomal abnormality. One SARS-CoV-2-positive woman delivered an infant who showed perinatal asphyxia and who required immediate mechanical ventilation and oxygen supplementation during the first 3 days of life. This woman and her infant are referred to collectively herein as Patient 1 . Neurological assessment was pathological (Sarnat score 1-2) and the neonate was treated with systemic hypothermia for 72 hours. Several episodes of interrupted electrical activity were noted at cerebral function monitoring, with an episode of seizures on day 7 that required administration of phenobarbital. Peripheral hyperexcitability, weak suction, and excessive crying were noted. All symptoms progressively improved, with spontaneous breathing on day 8 of life and drug stopped on day 17. The neonate was discharged at 27 days of life in good clinical condition (Figure 1A).

SARS-CoV-2 testing results of neonatal throat swabs of all newborns were negative, including multiple time points of Patient 1 (at birth, at 10 and 20 days of life, and at discharge) 


\section{Table 1. Characteristics of the study cohorts at admission to the emergency department and during hospitalization}

\begin{tabular}{|c|c|c|}
\hline Variable & COVID $^{+}$ & COVID- \\
\hline No. of patients & $21(57)$ & $16(43)$ \\
\hline Age, years & $33(24-37)$ & $35(32-38)$ \\
\hline Cestational age at diagnosis, gw + days (range) & $37+3(35-38 ; 2-6)$ & $39+1(39-40 ; 0-3)$ \\
\hline \multicolumn{3}{|l|}{ Ethnicity } \\
\hline White & $11(52)$ & $13(81)$ \\
\hline Hispanic & $7(33)$ & $2(12)$ \\
\hline Asian & $3(14)$ & $1(6)$ \\
\hline Smoker & $1(0)$ & $0(0)$ \\
\hline $\mathrm{BMI}\left(\mathrm{kg} / \mathrm{m}^{2}\right)$ & $27(24-32)$ & $25(24-27)$ \\
\hline \multicolumn{3}{|l|}{ Comorbidities } \\
\hline Asthma & $1(5)$ & $0(0)$ \\
\hline Hypothyroidism & $1(5)$ & $0(0)$ \\
\hline Anemia & $1(5)$ & $0(0)$ \\
\hline Adrenogenital syndrome & $1(5)$ & $0(0)$ \\
\hline Sclerodermia & $0(0)$ & $1(6)$ \\
\hline \multicolumn{3}{|l|}{ Pregnancy complications } \\
\hline Gestational diabetes & $2(10)$ & $3(19)$ \\
\hline Gestational cholestasis & $1(5)$ & $0(0)$ \\
\hline IUGR & $0(0)$ & $1(6)$ \\
\hline AF anomalies & $2(10)$ & $0(0)$ \\
\hline PROM & $2(10)$ & $2(12)$ \\
\hline pPROM & $1(5)$ & $1(6)$ \\
\hline Fetal complications & $2(10)$ & $0(0)$ \\
\hline \multicolumn{3}{|c|}{ Indication for nasopharyngeal swab at ED presentation } \\
\hline Screening & $9(43)$ & $15(94)$ \\
\hline Signs and symptoms & $12(57)$ & $1(6)$ \\
\hline Fever & $7(33)$ & $0(0)$ \\
\hline Cough & $8(38)$ & $1(6)$ \\
\hline Cold & $4(19)$ & $0(0)$ \\
\hline Dyspnea & $4(19)$ & $1(6)$ \\
\hline Myalgia & $2(10)$ & $0(0)$ \\
\hline Fatigue & $2(10)$ & $0(0)$ \\
\hline Anosmia & $1(5)$ & $0(0)$ \\
\hline Ageusia & $1(5)$ & $0(0)$ \\
\hline Altered mental status & $1(5)$ & $0(0)$ \\
\hline \multicolumn{3}{|c|}{ Indication for nasopharyngeal swab during hospitalization } \\
\hline Interstitial pneumonia & $7(33)$ & $0(0)$ \\
\hline Pulmonary thromboembolism & $2(10)$ & $0(0)$ \\
\hline Respiratory failure & $2(10)$ & $0(0)$ \\
\hline Transfer to ICU & $2(10)$ & $1(6)$ \\
\hline \multicolumn{3}{|l|}{ Respiratory support } \\
\hline Oxygen therapy & $4(19)$ & $1(6)$ \\
\hline NIV & $3(14)$ & $0(0)$ \\
\hline IV & $1(5)$ & $1(6)$ \\
\hline \multicolumn{3}{|l|}{ Pharmacological therapy } \\
\hline LMWH & $14(67)$ & $6(37)$ \\
\hline Before delivery & $2(10)$ & $0(0)$ \\
\hline After delivery & $14(67)$ & $6(37)$ \\
\hline HCQ & $6(29)$ & $0(0)$ \\
\hline \multicolumn{3}{|c|}{$\begin{array}{l}\text { Dichotomous variables were expressed as count (percentage) and } \\
\text { continuous variables as median (interquartile range). gw, gestational week } \\
\text { IUCR: intrauterine growth restriction; AF, amniotic fluid; PROM, premature } \\
\text { rupture of membrane; pPROM, preterm premature rupture of membrane; } \\
\text { NIV, noninvasive ventilation; IV, invasive ventilation; ICU, intensive care } \\
\text { unit; LMWH, low-molecular-weight heparin; HCQ, hydroxycloroquine. }\end{array}$} \\
\hline
\end{tabular}

Consistent with previous findings $(8,14)$, placental histopathology did not differ substantially between SARS-CoV-2-positive and -negative women, with alterations indicative of maternal inflammation rather than direct viral infection (Supplemental Table 1; supplemental material available online with this article; https://doi.org/10.1172/JCI145427DS1). One exception was that Patient 1 displayed unique features of severe placental injury with massive fibrin deposition and necrosis of the syncytiotrophoblast layer of the villi (Figure 1B and Supplemental Figure 1).

SARS-CoV-2 RNA could be detected in the placenta of 10 (47\%) SARS-CoV-2-positive women by PCR. Presence of the virus was not associated with any maternal or neonatal features (Supplemental Table 2).

The severity of SARS-CoV-2-mediated placental pathological changes might be directly related to viral load. The median viral load (expressed as PCR cycle thresholds) among SARS-CoV-2positive placentas without severe injury was 32 (IQR, 31-35). By contrast, the placenta with severe injury (from Patient 1) showed very high viral load $(\mathrm{Ct}=14)$, comparable to postmortem lung specimens from SARS-CoV-2-positive patients used as controls (Supplemental Tables 3 and 4). Furthermore, SARS-CoV-2 RNAISH revealed an intense positivity of perivillous trophoblast with extensive apoptosis of the infected cellular compartment in the placenta from Patient 1 not found in the other placentas and similar to what was observed in lung samples (Figure 1, B and C, and Supplemental Figures 2 and 3).

Previous studies have shown that SARS-CoV-2 induces a host inflammatory response in the upper respiratory tract that may be a primary driver of the signature pathology observed in patients with COVID-19 (18). Consistent with this, comparative gene expression analysis showed that all placentas clustered together irrespective of SARS-CoV-2 status, except for the 2 independent samples from the placenta from Patient 1 , which segregated with lung specimens in a distinct cluster (Figure 2, A and B). Signature enrichment and differential expression analyses showed that genes implicated in innate antiviral immunity, chemotactic and inflammatory response, and adaptive response were highly expressed in the placenta from Patient 1 and the infected lungs, compared with the rest of the placenta samples (Figure 2C and Supplemental Figure 4). Volcano plots allowed us to identify significant gene expression differences between infected lung and placenta as well as placentas with intermediate and low inflammation (Supplemental Figure 4, B and C).

Despite no vertical transmission being observed in this series, our data show that SARS-CoV-2 can be detected in placental tissue by PCR in almost half of pregnant women infected during the third trimester. This is higher than what has been reported so far in published case series with positivity rates ranging from $6.5 \%$ to $27.3 \%$ (14-17) and from the $7.7 \%$ rate emerging from a systematic review (19). The reasons for this discrepancy may include cohort geography, number of samples analyzed, sampling methodologies, and techniques used for viral RNA detection, among others. Importantly, in our series the positivity rate dropped to $5 \%$ when using a less sensitive technique (such as in RNA-ISH, Supplemental Table 4), which is in line with percentages reported in studies using a similar approach $(14,17)$. 
Table 2. Maternal and neonatal outcome at delivery

\begin{tabular}{|c|c|c|}
\hline Variable & COVID $^{+}$ & COVID- $^{-}$ \\
\hline No. of patients & $21(57)$ & $16(43)$ \\
\hline Gestational age at delivery (gw+days) & $38+3(36-39 ; 2-5)$ & $39+3(39-40 ; 0-3)$ \\
\hline Symptom onset to delivery (days) & $9(4-11)$ & - \\
\hline Confirmed diagnosis to delivery (days) & $2(1-6)$ & - \\
\hline Premature delivery (< $37 \mathrm{gw})$ & $6(29)$ & $3(19)$ \\
\hline \multicolumn{3}{|l|}{ Mode of delivery } \\
\hline Vaginal delivery & $12(67)$ & $5(31)$ \\
\hline Cesarean section & $9(43)$ & $10(62)$ \\
\hline Spontaneous labor & $5(33)$ & $5(31)$ \\
\hline Labor by induction & $7(67)$ & $1(6)$ \\
\hline \multicolumn{3}{|l|}{ Maternal indication for labor induction } \\
\hline SARS-CoV-2 infection & $3(14)$ & - \\
\hline \multicolumn{3}{|l|}{ Obstetrical indication for labor induction } \\
\hline AF abnormalities & $2(10)$ & $0(0)$ \\
\hline Fetal malformation & $1(5)$ & $0(0)$ \\
\hline Therapeutic miscarriage & $1(5)$ & $0(0)$ \\
\hline Macrosomia & $0(0)$ & $1(6)$ \\
\hline Suspected chorioamniositis & $0(0)$ & $0(0)$ \\
\hline \multicolumn{3}{|l|}{ Type of cesarean section } \\
\hline Elective & $4(44)$ & $4(40)$ \\
\hline Emergency & $5(55)$ & $6(60)$ \\
\hline \multicolumn{3}{|l|}{ Indication for cesarean section } \\
\hline Maternal request & $2(10)$ & $1(6)$ \\
\hline Previous cesarean section & $2(10)$ & $1(6)$ \\
\hline IUGR & $0(0)$ & $1(6)$ \\
\hline Uterine malformation & $0(0)$ & $1(6)$ \\
\hline Maternal clinical conditions & $0(0)$ & $1(6)$ \\
\hline Fetal distress & $2(10)$ & $4(25)$ \\
\hline Symptomatic SARS-CoV-2 infection & $1(5)$ & - \\
\hline Symptomatic SARS-CoV-2 infection and suspected PE & $1(5)$ & - \\
\hline Symptomatic SARS-CoV-2 infection and previous CS & $1(5)$ & - \\
\hline \multicolumn{3}{|l|}{ Neonatal outcome } \\
\hline Apgar, minute 1 & $9(9-9)$ & $9(8-9)$ \\
\hline Apgar, minute 5 & $10(10-10)$ & $10(9-10)$ \\
\hline Newborn birthweight (g) & $3020(2541-3359)$ & $3030(2810-3430)$ \\
\hline Placental weight (g) & $490(400-565)$ & $500(470-700)$ \\
\hline Neonatal arterial pH & $7.3(7.21-7.34)$ & $7.2(7.17-7.25)$ \\
\hline
\end{tabular}

Dichotomous variables were expressed as count (percentage) and continuous variables as median (interquartile range). gw, gestational week; AF, amniotic fluid; IUGR, intrauterine growth restriction; PE, pulmonary embolism.

The low viral tissue load found in the placentas was unable to induce an inflammatory response, as supported by maternal and neonatal clinical outcomes, placenta histology, and molecular data that did not differ according to SARS-CoV-2 infection. These results suggest that the placenta may not be a preferential target for SARS-CoV-2 infection, possibly due to the minimal expression in the placenta of canonical cell-entry mediators for the virus (14, $20)$. This idea supports previous findings regarding mother and infant health $(7,15,21)$.

Although reassuring, our study also provides solid evidence that, although it happens only occasionally, SARS-CoV-2 can infect the placenta with high viral load and lead to a function- al impairment of the organ that can ultimately impact neonatal outcome even in the absence of vertical transmission. As PCR detection of the virus in placental homogenates may be contaminated by maternal cells, cellular evidence of viral infection of villous trophoblasts using ISH or immunohistochemistry represents a definitive demonstration of placental infection. In our series, one case showed diffuse SARS-CoV-2 positivity of perivillous trophoblastic cells by ISH, which was associated with increased apoptosis and massive villous infarction not found in the other placentas. The placental transcriptional response induced by a high viral load of SARS-CoV-2 is characterized by the overexpression of genes implicated in innate antiviral immunity and strong chemotactic and inflammatory responses, such as CXCL9, CXCL10, CXLC11, CCL2, CCL7, IL6, IL21R, CD8A, GZMA, PRF1, CD68, and CD163. Interestingly, this transcriptional pattern is similar to the one observed in the SARS-CoV-2infected lungs, with some tissue-specific differences worthy of further investigation. Hence, an important observation of this study is that even though there was a high SARS-CoV-2 titer in the placenta, no virus was detected in the neonate. This result suggests that the placenta may provide an effective protective barrier against the virus, inhibiting the intrauterine transmission to the fetus. However, viral infection of the placenta could trigger a fetal inflammatory response, which in turn can cause organ damage and downstream developmental deficiencies, as previously shown for other pathogens (22-25). Indeed, viral crossing of the placenta may be the exception rather than the rule. Examples of viral infections that are unable to reach the fetus but might have a detrimental effect on the pregnancy are HIV, hepatitis B, varizella zoster virus, and influenza, among others $(22,26,27)$. Many reasons could be related to the high viral infection present in the placenta from Patient 1. Most likely, a high viral load present in the blood of the mother might be the cause of its access to the placenta. Moreover, a potential differential virus receptor expression in the placental tissue or an immunocompromised status of the mother might also be responsible for the high viral load observed in the placenta.

Ours is a proof-of-concept study and further studies are warranted to fully estimate the incidence of this phenomenon. In the published literature, there are only 13 proven cases of direct placenta infection among more than 250 neonates from SARS-CoV-2-positive pregnant women reported so far. Newborns were asymptomatic in 9 cases $(11,14-16)$, whereas in 4 cases they required admission to the neonatal intensive care unit for neurological disorders at birth (10), repeated episodes of hypoglycemia (12), feeding difficulties (12), and interstitial pneumonia (17). One case required termination of pregnancy to reduce the risk of maternal morbidity (13). Eight newborns tested positive for SARS-CoV-2 by PCR on nasopharyngeal swab. Two newborns tested positive in umbilical cord plasma (15). In our study, we did not have access to umbilical cord blood for anti-SARS-CoV-2 antibodies testing, thus we cannot completely exclude a possible intrauterine exposure of the fetus.

The observation that SARS-CoV-2 can infect the placenta to impact fetal development is of great relevance and indicates that further and more strict monitoring of pregnancy should be undertaken in SARS-CoV-2-infected mothers. Our data link the 
A Patient 1 clinical history

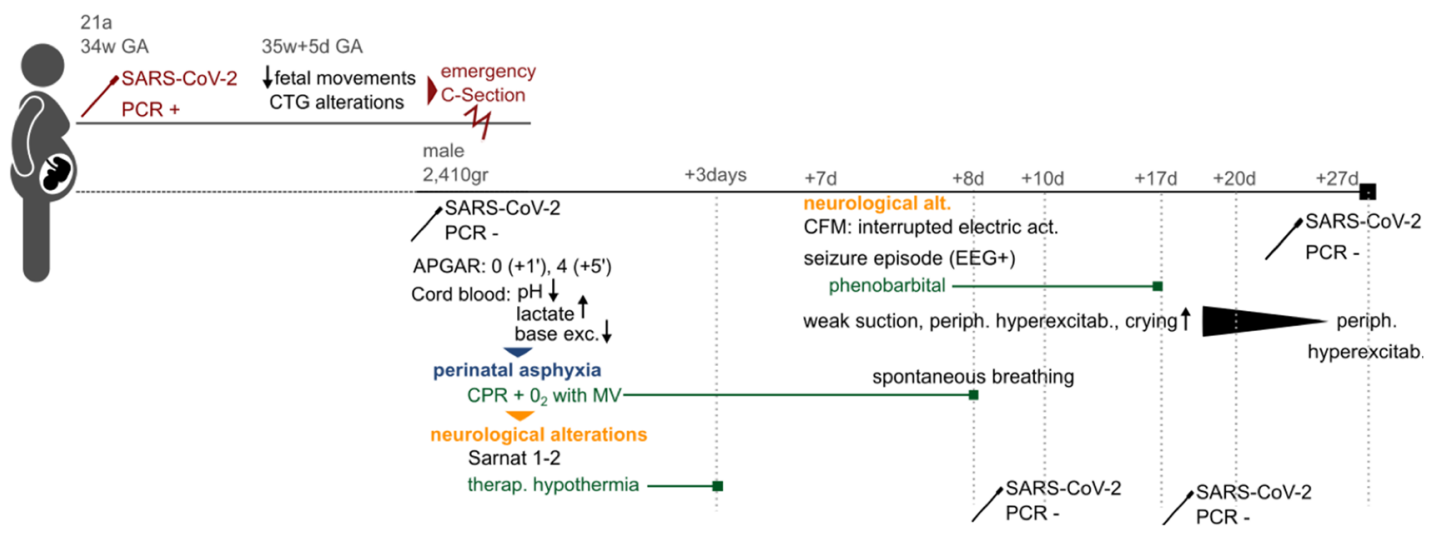

B Histopathological and molecular features of the placenta from Patient 1

H\&E

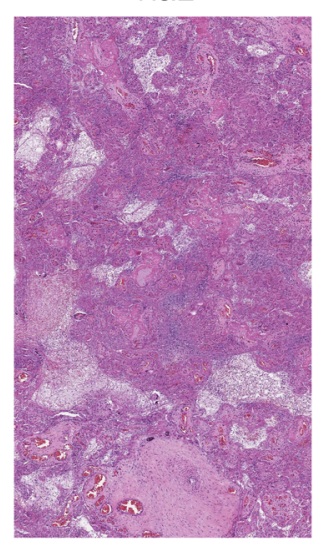

SARS-CoV-2 RNA-ISH

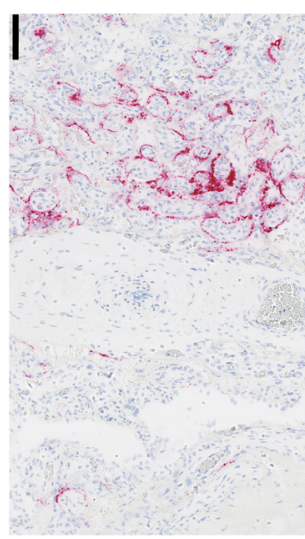

Cleaved caspase 3

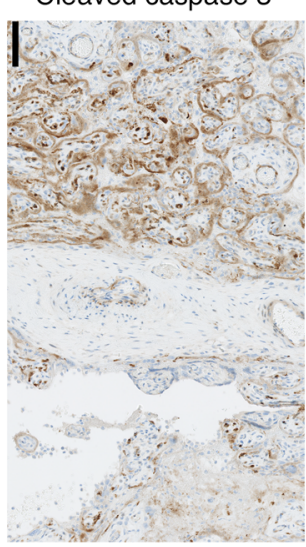

Figure 1. Examination of SARS-CoV-2 in Patient 1. (A) Case timeline. (B) Histopathological and molecular features of the placenta with severe injury. Massive fibrin deposition associated with syncytiotrophoblast layer necrosis and ghost villi (left, H\&E staining). Original magnification, $\times 10$. See also Supplemental Figure 1. Placental SARS-CoV-2 in situ hybridization reveals an intense positivity (red staining) of perivillous trophoblastic cells (middle, RNA-ISH). Marked peri-villous trophoblastic cells apoptosis outlined by cleaved caspase-3 brown staining (right, immunohistochemistry). Scale bar: $100 \mu \mathrm{m}$.

severity of SARS-CoV-2-induced placental damage with detrimental neonatal outcome at birth with no related vertical transmission of the infection. Although rare, early detection of this phenomenon is important to adopt specific therapeutic strategies to prevent neonatal complications.

\section{Methods}

Detailed methods are provided in the Supplemental Methods.

Patient data. Placentas from 37 pregnant women admitted between March 12, 2020, and April 23, 2020, at Fondazione IRCCS Ca' Granda - Ospedale Maggiore Policlinico (Milan, Italy) were included in the study. All women were tested at admission for SARSCoV-2 infection by nasopharyngeal swab PCR. Formalin-fixed paraffin-embedded (FFPE) placental tissue samples were collected from all women included in the study. Lung samples from SARS-CoV-2positive patients were collected postmortem at the Department of Pathology, University of Basel (Switzerland).

$R T-P C R$. Quantitative RT-PCR for SARS-CoV-2 in placenta and lung tissue samples were performed using the Allplex
2019-nCoV Assay (Seegene Inc) and TaqMan 2019-nCoV Assay Kit-v2 (Thermo Fisher Scientific) according to the manufacturer's instructions.

In situ analyses. Viral RNA was visualized in FFPE tissue sections using the RNAscope (Advanced Cell Diagnostics USA) in situ hybridization assay. Apoptosis was analyzed by IHC using the anti-cleaved caspase-3 (Asp175) rabbit polyclonal antibody from Cell Signaling Technologies (catalog 9661).

Gene expression. RNA expression profiling was performed using Nanostring, with a panel of 90 genes built in-house containing the genes specified in the 3 gene signatures of interest: cytotoxic cells, macrophages, and IFN- $\gamma$ (Supplemental Table 5).

Study approval. The study was approved by the ethics committees of Fondazione IRCCS Ca' Granda-Ospedale Maggiore Policlinico Milano and Northwest and Central Switzerland (EKNZ).

\section{Author contributions}

FMC, RE, and LP collected, analyzed, and interpreted data and wrote the manuscript. GAC, AR, CP, EF, GL, AMF, SF, EI, MP, 
A

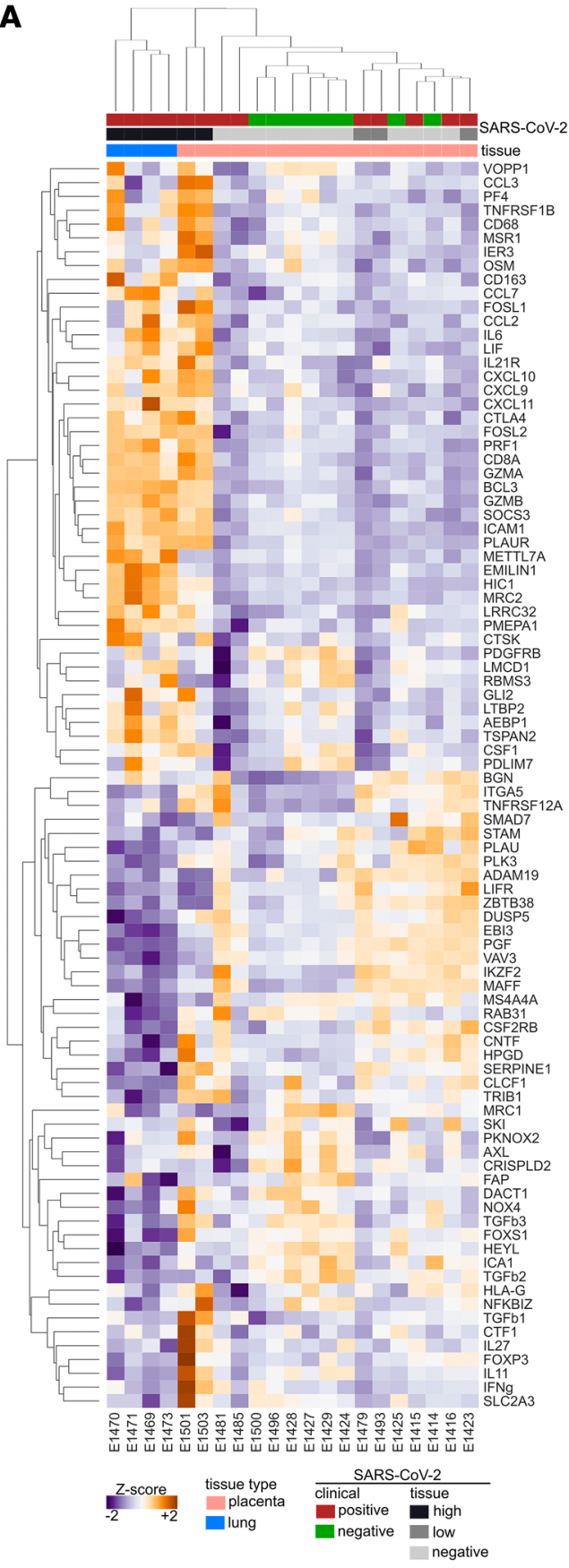

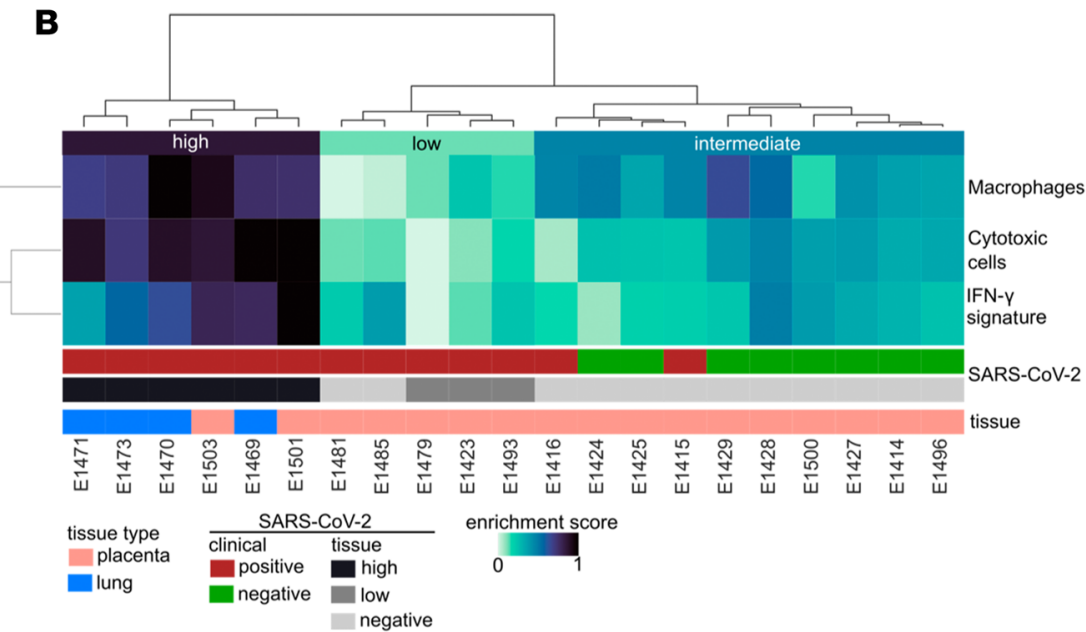

C

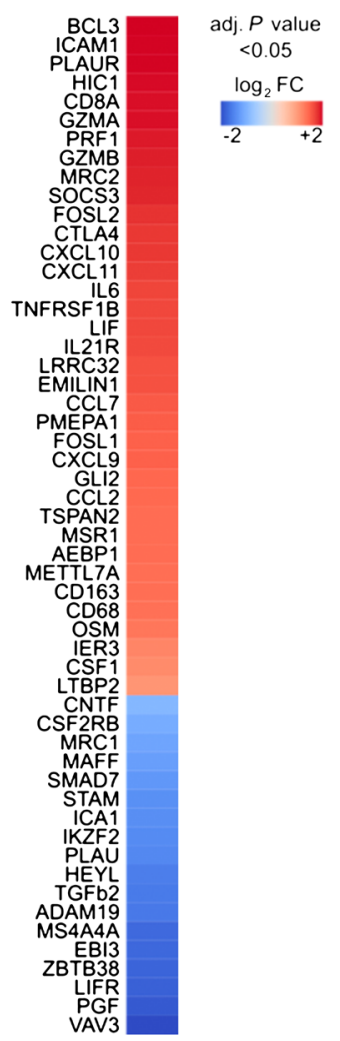

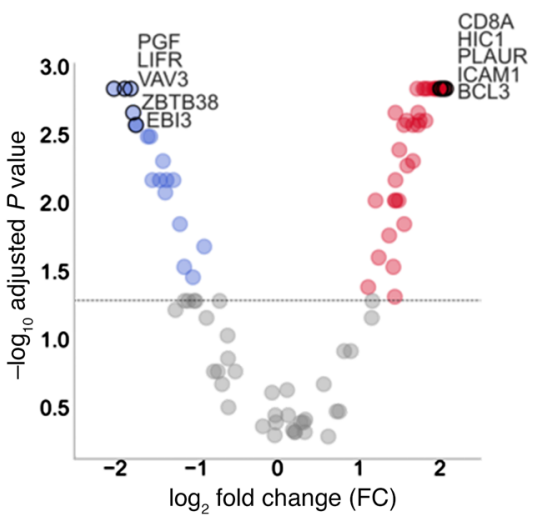

Figure 2. Gene expression analyses. (A) Heatmap representing the expression values (z-scaled) of all genes in the Nanostring panel, excluding housekeeping genes ( $n=90$; rows), across lung and placenta samples $(n=21$; columns). (B) Heatmap representing the enrichment scores of cytotoxic cells, macrophages, and IFN- $\gamma$ signature (rows) in the placenta and lung cohorts $(n=21)$. E1501 and E1503 are 2 independent samples of severe injured placenta from Patient 1. On top, the resulting dendrogram of sample aggregation (columns) according to the expression values of all genes (A) and enrichment of the 3 signatures (B) are shown. On the left, the dendrogram of gene/signature aggregation (rows) is shown. Sample tissue and SARS-CoV-2 status are highlighted as colored heatmap annotations. (C) Differential genes expression analysis comparing severe injured placenta from Patient 1 ( $n=2$ ) and lung samples $(n=6)$ with the rest of the placental samples $(n=15)$. In the volcano plot (right), each dot represents a gene and is colored according to Mann Whitney $U$ test results and the $\log _{2}$ fold change (FC). Significantly overexpressed genes (adjusted $P<0.05$ and FC $>0$ ) are colored red and significantly underexpressed genes (adjusted $P<0.05$ and $\mathrm{FC}<0$ ) are colored blue; nonsignificant differentially expressed genes are colored gray. A dashed line represents the significance threshold, adjusted $P=0.05$. The top 5 significantly over- and underexpressed genes are highlighted. Column heatmap representing all significant results (adjusted $P<0.05$ ) of the differential expression analysis in (B) (left). The column represents the log ${ }_{2}$ FC.

MM, AT, and LT collected and analyzed data. AA and GS collected, analyzed, and interpreted data. LA collected and analyzed data and created figures. SS performed a literature search and analyzed data. CRP analyzed and interpreted data and created figures. JS and PN designed the study, analyzed and interpreted data, wrote the manuscript, and created figures. 


\section{Acknowledgments}

The authors acknowledge the Cellex Foundation for providing research facilities and equipment. The study was undertaken with the support of a COVID-19 Direcció General de Recerca i Innovació en Salut grant (Generalitat de Catalunya), the Fundación Asociación Española contra el Cáncer (AECC), the Ramón Areces Foundation, the Instituto de Salud Carlos III, the Fondo de Investigaciones Sanitarias (PI19/00318), and the Juan de la Cierva formación fellowship (to CRP). AT was supported by the Botnar Research Centre for Child Health (BRCCH) at the University of Basel.

Address correspondence to: Paolo Nuciforo or Joan Seoane, Vall d'Hebron Institute of Oncology (VHIO), Centre Cellex, C/Natzaret, 115-117, Barcelona 08035, Spain. Phone: 34.93.254.34.50 ext. 8626; Email: pnuciforo@vhio.net (PN); jseoane@vhio.net (JS).
1. Liu W, et al. Clinical characteristics of 19 neonates born to mothers with COVID-19. Front Med. 2020;14(2):193-198.

2. Zhu H, et al. Clinical analysis of 10 neonates born to mothers with 2019-nCoV pneumonia. Transl Pediatr. 2020;9(1):51-60.

3. Chen $\mathrm{H}$, et al. Clinical characteristics and intrauterine vertical transmission potential of COVID-19 infection in nine pregnant women: a retrospective review of medical records. Lancet. 2020;395(10226):809-815.

4. Yan J, et al. Coronavirus disease 2019 in pregnant women: a report based on 116 cases. Am JObstet Gynecol. 2020;223(1):111.e1-111.e14.

5. Schwartz DA. An analysis of 38 pregnant women with COVID-19, their newborn infants, and maternal-fetal transmission of SARS-CoV-2: maternal Coronavirus infections and pregnancy outcomes. Arch Pathol Lab Med. 2020;144(7):799-805.

6. Lamouroux A, et al. Evidence for and against vertical transmission for severe acute respiratory syndrome coronavirus 2. Am JObstet Gynecol. 2020;223(1):91.e1-91.e4.

7. Raschetti R, et al. Synthesis and systematic review of reported neonatal SARS-CoV-2 infections. Nat Commun. 2020;11(1):5164.

8. Shanes ED, et al. Placental pathology in COVID19. Am J Clin Pathol. 2020;154(1):23-32.

9. Algarroba GN, et al. Visualization of severe acute respiratory syndrome coronavirus 2 invading the human placenta using electron microscopy. Am J Obstet Gynecol. 2020;223(2):275-278.

10. Vivanti AJ, et al. Transplacental transmis- sion of SARS-CoV-2 infection. Nat Commun. 2020;11(1):3572.

11. Patanè L, et al. Vertical transmission of coronavirus disease 2019: severe acute respiratory syndrome coronavirus 2 RNA on the fetal side of the placenta in pregnancies with coronavirus disease 2019-positive mothers and neonates at birth. Am JObstet Gynecol MFM. 2020;2(3):100145-100145.

12. Kirtsman M, et al. Probable congenital SARSCoV-2 infection in a neonate born to a woman with active SARS-CoV-2 infection. CMAJ. 2020;192(24):E647-E650.

13. Hosier H, et al. SARS-CoV-2 infection of the placenta. J Clin Invest. 2020;130(9):4947-4953.

14. Hecht JL, et al. SARS-CoV-2 can infect the placenta and is not associated with specific placental histopathology: a series of 19 placentas from COVID-19-positive mothers. Mod Pathol. 2020;33(11):2092-2103.

15. Fenizia C, et al. Analysis of SARS-CoV-2 vertical transmission during pregnancy. Nat Commun. 2020;11(1):5128.

16. Penfield CA, et al. Intrauterine vertical transmissibility of SARS-CoV-2: the evidence is evolving. Am JObstet Gynecol MFM. 2020;2(4):100227-100227.

17. Facchetti F, et al. SARS-CoV2 vertical transmission with adverse effects on the newborn revealed through integrated immunohistochemical, electron microscopy and molecular analyses of placenta. EBioMedicine. 2020;59:102951.

18. Lee JS, et al. Immunophenotyping of COVID-19 and influenza highlights the role of type I interferons in development of severe COVID-19. Sci
Immunol. 2020;5(49):eabd1554.

19. Kotlyar AM, et al. Vertical transmission of coronavirus disease 2019: a systematic review and meta-analysis. Am JObstet Gynecol. 2021;224(1):35-53.

20. Pique-Regi R, et al. Does the human placenta express the canonical cell entry mediators for SARS-CoV-2? Elife. 2020;9:58716.

21. Stowe J, et al. Stillbirths during the COVID-19 pandemic in England, April-June 2020. JAMA. 2021;325(1):86-87.

22. Cardenas I, et al. Viral infection of the placenta leads to fetal inflammation and sensitization to bacterial products predisposing to preterm labor. J Immunol. 2010;185(2):1248-1257.

23. Deverman BE, Patterson PH. Cytokines and CNS development. Neuron. 2009;64(1):61-78.

24. Meyer U, et al. A review of the fetal brain cytokine imbalance hypothesis of schizophrenia. Schizophr Bull. 2009;35(5):959-972.

25. Golan HM, et al. Specific neurodevelopmental damage in mice offspring following maternal inflammation during pregnancy. Neuropharmacology. 2005;48(6):903-917.

26. Connor EM, et al. Reduction of maternal-infant transmission of human immunodeficiency virus type 1 with zidovudine treatment. Pediatric AIDS clinical trials group protocol 076 study group. NEngl J Med. 1994;331(18):1173-1180.

27. Arrivé E, Dabis F. Prophylactic antiretroviral regimens for prevention of mother-to-child transmission of HIV in resource-limited settings. Curr Opin HIV AIDS. 2008;3(2):161-165. 